\title{
Logística de medicamentos na palma da mão: Aplicativo logmed
}

\author{
Drug logistics in the palm of the hand: Logmed application \\ La logística de drogas en la palma de la mano: Aplicación logmed
}

Recebido: 19/06/2021 | Revisado: 25/06/2021 | Aceito: 28/06/2021 | Publicado: 13/07/2021

Suellen de Abreu Soares
ORCID: https://orcid.org/0000-0002-3429-0881
E-mail: suellen@ufrj.br
Patrícia Gonçalves Trentin
Universidade Federal do Estado do Rio de
Paneiro, Bril
ORCID: https://orcid.org/0000-0002-6911-6031
Hospital Universitário Gaffrée e Guinle, Brasil
E-mail: patricia.trentin@ebserh.gov.br
Matheus da Silva Tavares Henriques
ORCID: https://orcid.org/0000-0003-0197-9778
Hospital Central da Aeronáutica, Brasil
E-mail: matheus-sth@hotmail.com
Paulo Sergio Marcellini
Paulo
ORCID: https://orcid.org/0000-0001-5618-5348
E-mail: marcellinips@yahoo.com
Universidade Federal do Estado do Rio de Janeiro, Brasil
ORCID: https://orcid.org/0000-0002-8981-4662
E-mail: alanxcampos@gmail.com
Universidade Federal do Estado do Rio de Janeiro, Brasil
Michele Lúcia de Aguiar Mitsuyasu
ORCID: https://orcid.org/0000-0001-5774-2097
Universidade Federal Fluminense, Brasil
E-mail: michele.mitsuyasu@gmail.com
Camila Carvalho Lopes
ORCID: https://orcid.org/0000-0001-5260-3987
Hospital Central da Aeronáutica, Brasil
E-mail: camilaccl@fab.mil.br
Catiane Menezes Duarte Vieira
ORCID: https://orcid.org/0000-0003-4924-210X
Universidade Federal do Rio de Janeiro, Brasil
E-mail: catianeduarte@gmail.com

\section{Resumo}

Objetivo: Descrever a criação de um aplicativo no sistema operacional Android@ que disponibilize informações para a otimização do processo de gestão das aquisições públicas de medicamentos. Métodos: Foi realizado um estudo exploratório experimental, dividido nas seguintes etapas: revisão da legislação e literatura, desenvolvimento do protótipo através de um website e avaliação de usabilidade por meio de questionário específico desenvolvido pela Universidade Federal de Santa Catarina (GQS/UFSC) - questionário MATcH. Resultado: Definição dos documentos e informações a serem utilizados no aplicativo, de acordo com a pesquisa bibliográfica, demonstração dos recursos disponíveis no aplicativo, e aplicação do questionário MATcH, para validação de usabilidade do protótipo. Conclusão: constatou-se com este estudo que o Logmed é uma ferramenta útil, para acompanhamento do processo de gestão da aquisição de medicamentos, visando evitar o desabastecimento e os consequentes prejuízos na assistência.

Palavras-chave: Assistência farmacêutica; Proposta de concorrência; Serviço de farmácia hospitalar; Serviço hospitalar de compras; Acesso a medicamentos essenciais e tecnologias em saúde.

\begin{abstract}
Objective: Describe the creation of an application on the Android® operating system that provides information for optimizing the management process of public procurement of medicines. Methods: An exploratory experimental study was carried out, divided into the following steps: review of legislation and literature, development of the prototype through a website and usability assessment through a specific questionnaire developed by the Federal University of Santa Catarina (GQS/UFSC) - questionnaire MATCH. Result: Definition of documents and information to be used in the application, according to the bibliographic research, demonstration of the resources available in the application, and application of the MATcH questionnaire, to validate the prototype's usability. Conclusion: it was found with this study that Logmed is a useful tool for monitoring the process of managing the acquisition of medicines, aiming to avoid shortages and the consequent damages in care.
\end{abstract}


Keywords: Pharmaceutical services; Competitive bidding; Pharmacy service hospital; Purchasing hospital; Access to essential medicines and health technologies.

\begin{abstract}
Resumen
Objetivo: Describir la creación de una aplicación en el sistema operativo Android® que brinde información para optimizar el proceso de gestión de la contratación pública de medicamentos. Métodos: Se realizó un estudio experimental exploratorio, dividido en los siguientes pasos: revisión de legislación y literatura, desarrollo del prototipo a través de un sitio web y evaluación de usabilidad a través de un cuestionario específico desarrollado por la Universidad Federal de Santa Catarina (GQS / UFSC) - cuestionario MATcH. Resultado: Definición de documentos e información a utilizar en la aplicación, de acuerdo con la búsqueda bibliográfica, demostración de los recursos disponibles en la aplicación y aplicación del cuestionario $\mathrm{MATcH}$, para validar la usabilidad del prototipo. Conclusión: con este estudio se encontró que Logmed es una herramienta útil para monitorear el proceso de gestión de la adquisición de medicamentos, con el objetivo de evitar desabastecimientos y los consiguientes perjuicios en la atención.

Palabras clave: Servicios farmacéuticos; Propuestas de licitación; Servicio de farmacia en hospital; Departamento de compras en hospital; Acceso a medicamentos esenciales y tecnologías sanitarias.
\end{abstract}

\title{
1. Introdução
}

No serviço público as aquisições tanto de bens materiais (incluindo os medicamentos) quanto de serviços, são feitas por licitações, conforme preconizado pela Lei no 8.666 de 21 de junho de 1993. (BRASIL,1993).

O Tribunal de Contas da União define licitação como o procedimento administrativo formal em que a Administração Pública convoca, por meio de condições estabelecidas em ato próprio (edital ou convite), empresas que desejam apresentar propostas para o oferecimento de bens e serviços para a Administração Pública. O objetivo é proporcionar maior economia para a Administração Pública selecionando a proposta mais vantajosa, assegurando oportunidades iguais a todos os interessados e possibilitando o comparecimento ao certame do maior número possível de Empresas concorrentes.

Diante desse cenário torna-se imprescindível um bom gerenciamento da cadeia logística de medicamentos a fim de viabilizar o uso correto dos recursos, a programação e aquisição de produtos em quantidade e qualidade adequadas, garantindo que estes cheguem ao estoque no momento certo, na quantidade correta, e adequados para uso.

Os gastos crescentes no setor da saúde são alvo de preocupação, tanto nas empresas privadas, quanto nas instituições públicas. As causas estão centradas na longevidade da população, incorporações tecnológicas, novos medicamentos, equipamentos e materiais médico-hospitalares, além de processos de compras inadequados e a lentidão na implantação de práticas já consagradas em outros setores da economia (Kumar \& Seema, 2017).

Apesar da singularidade e complexidade da cadeia de suprimentos de serviços de saúde, as oportunidades de melhorias são abundantes (Gonçalves et al., 2016).

A maior complexidade dos serviços médicos, impulsionada pelas inovações na ciência e tecnologia, traz a necessidade de novos modelos gerenciais para as organizações de saúde (Bridges, 2006).

O medicamento é um insumo estratégico de suporte às ações de saúde, cuja falta pode significar interrupções constantes no tratamento, o que afeta a qualidade de vida dos usuários e do sistema de saúde como um todo (BRASIL,2006., 2006).

$\mathrm{O}$ resultado imediato e de maior visibilidade da falta de um medicamento é o cancelamento ou adiamento de procedimentos e o consequente aumento dos riscos das enfermidades (Reis \& Perini, 2008).

O monitoramento e a gestão dos processos de aquisição são fundamentais para aprimorar a cadeia logística e intervir nos problemas. Eles devem ser baseados em instrumentos de aferição denominados indicadores. A falta desses indicadores, bem como de uma ferramenta para otimização da aquisição de medicamentos dificulta o processo de gerenciamento de medicamentos culminando nos desabastecimentos que impactam direta e indiretamente na qualidade da assistência à saúde do paciente.

Por relevância da pesquisa entende-se abrangência do tema e o impacto (aplicabilidade do resultado) no contexto científico, inovação, clínico, social, tecnológico, educacional ou econômico (Estrela, 2018). 
Constitui-se como objetivo geral desse trabalho, apresentar as etapas de criação de um aplicativo no sistema operacional Android® que disponibilize informações para a otimização do processo de gestão das aquisições públicas de medicamentos.

\section{Metodologia}

Os métodos qualitativos são aqueles nos quais é importante a interpretação por parte do pesquisador com suas opiniões sobre o fenômeno em estudo ( Pereira et al., 2018).

Sendo o principal instrumento da investigação, o observador pode recorrer aos conhecimentos e experiências pessoais como auxiliares no processo de compreensão e interpretação do fenômeno estudado (Lüdke, 2013).

Na pesquisa exploratória não se trabalha com a relação entre variáveis, mas com o levantamento da presença das variáveis e da sua caracterização quantitativa ou qualitativa.(Köche, José Carlos, 2011).

Foi realizado um estudo qualitativo descritivo exploratório, dividido nas seguintes etapas: revisão da legislação e literatura, desenvolvimento do protótipo através de um website e avaliação de usabilidade por meio de questionário específico desenvolvido pela Universidade Federal de Santa Catarina (GQS/UFSC) - questionário MATcH.

Por meio de buscas em sites governamentais em bases de dados Biblioteca Virtual em Saúde (BVS), Catálogo de Teses e Dissertações da Coordenação de Aperfeiçoamento de Pessoal de Nível Superior (CAPES), Scientific Electronic Library Online (SCIELO) e Google Acadêmmico, foi realizada uma revisão integrativa a partir dos Descritores em Ciências da Saúde (DeCs): "Armazenamento de medicamentos; Gestão de Materiais no Hospital". Foram estabelecidos como critérios de inclusão: produções científicas disponíveis na íntegra, bem como originais nos idiomas português e inglês. Foram excluídos os artigos que não estavam disponíveis integralmente na versão on-line ou cujo conteúdo divergia do assunto.

O aplicativo foi desenvolvido pelos autores por meio do site "Fábrica de Aplicativos", disponível no endereço eletrônico www.fabricadeaplicativos.com.br (Fabapp - Fabrica de Aplicativos, [s.d.])

Após a construção do aplicativo, foi aplicado Checklist para Avaliação da Usabilidade de Aplicativos para smartfones Touchscreen - questionário MATcH®, desenvolvido pelo grupo de qualidade em software da Universidade Federal de Santa Catarina (GQS/UFSC), disponível em http://match.inf.ufsc.br:90/. Essa é uma ferramenta de apoio à avaliação heurística como medida de usabilidade de aplicativos para smartphones (MATcH Checklist, [s.d.]).

Não foi necessário a apresentação deste estudo ao comitê de ética pois todos os dados utilizados na pesquisa são de domínio público.

\section{Resultados}

Um fluxograma (Figura 1) demonstra o processo de seleção dos estudos encontrados a fim de auxiliar na escolha dos resultados com maior nível de evidências.

Os dados foram analisados por meio de estatística descritiva, de modo que foi possível fazer a contabilização e a descrição dos dados encontrados. 
Figura 1 - Fluxograma de seleção da amostra de registros da pesquisa.
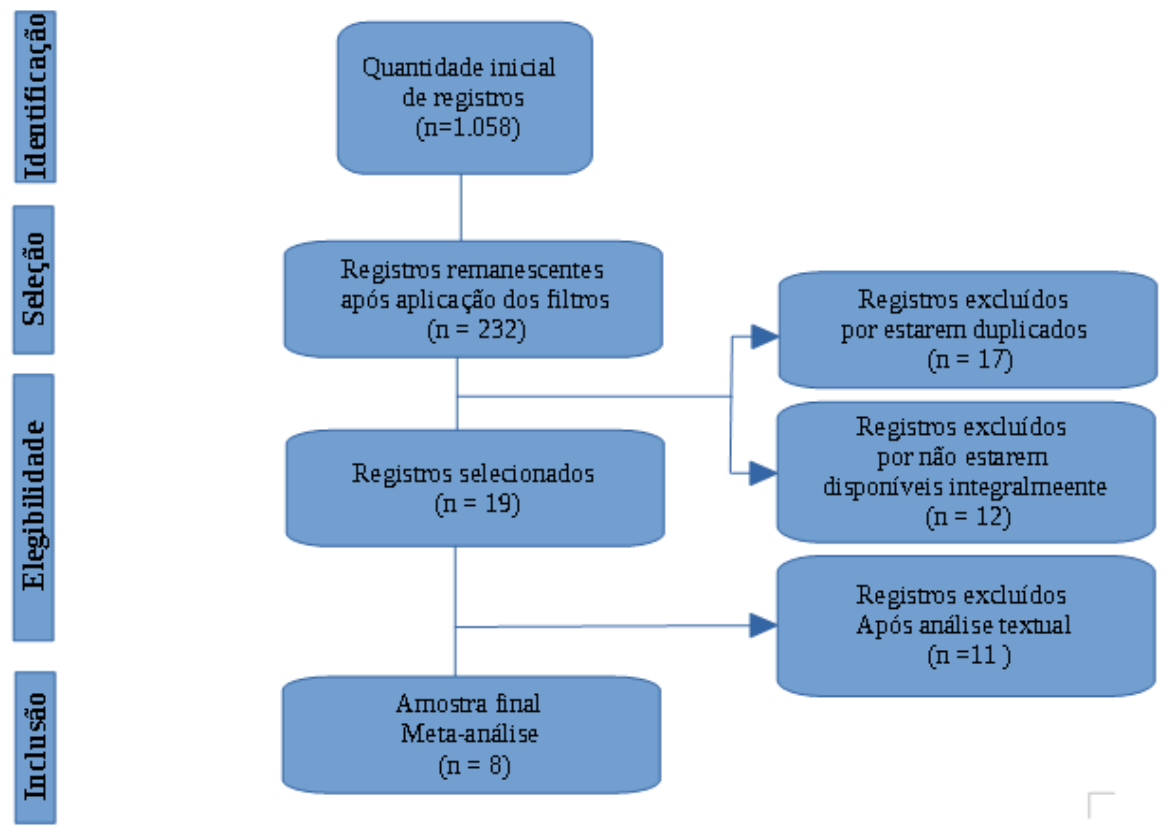

Fonte: Autores.

Foi realizada uma busca na loja virtual de aplicativos PlayStore ${ }^{\mathrm{TM}}$ a fim de se certificar do volume de ofertas de aplicativos com o mesmo objetivo proposto neste estudo. Para tanto, foram utilizados os termos: “compras públicas”, “gestão de fornecedores" e "gestão de compras". Não foram encontrados aplicativos semelhantes.

Os ícones foram definidos de forma a facilitar a sua identificação pelo usuário. A base da construção textual procedeu da legislação vigente relacionada às compras públicas Leis 8666/1993 e 10.520/2002, bem como das rotinas aplicadas à central de abastecimento farmacêutico do Hospital Central da Aeronáutica (Brasil,1993, [s.d.]; Brasil,2002, [s.d.]).

O conteúdo do aplicativo foi disposto por 5 telas principais, seguido de 6 abas secundárias. O mapa do aplicativo está mostrado na Figura 2.A. Para acesso à versão beta (versão de teste), o usuário deverá fotografar o QRCODE (Figura 2.B.) ou digitar o endereço eletrônico https://app.vc/logmed do aplicativo para ser direcionado à página para o download (App LogMed, [s.d.]). 
Figura 2. A. Mapa estruturado aplicativo LogMed.

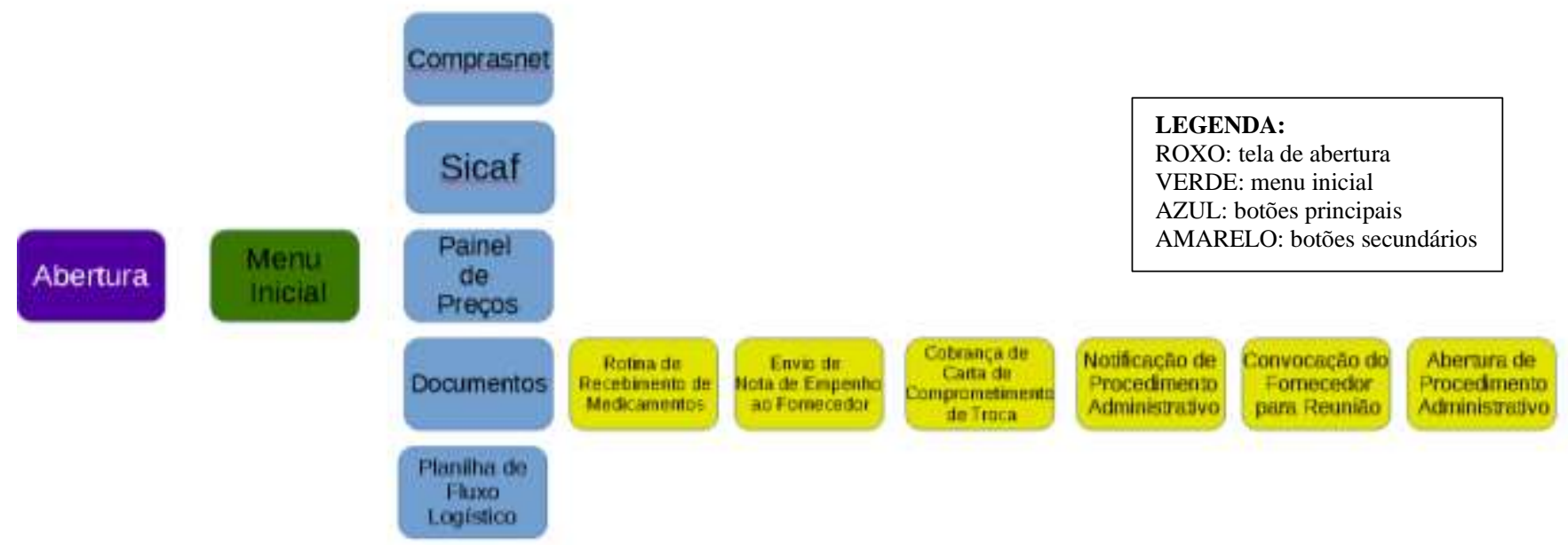

Figura 2. B. Ícone do botão QRCODE.

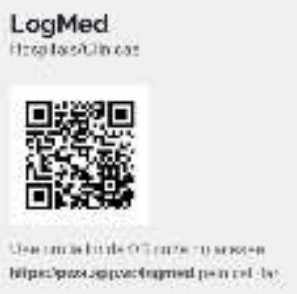

Fonte: Autores.

A tela de abertura apresenta um caminhão com um desenho de um comprimido (Figura 3.A.), fazendo alusão à logística de medicamentos. A imagem, de domínio público, foi extraída da internet. Após a tela de abertura encontra-se o menu inicial (Figura 3.B.).

Figura 3.A. Ícone da tela de abertura do aplicativo.

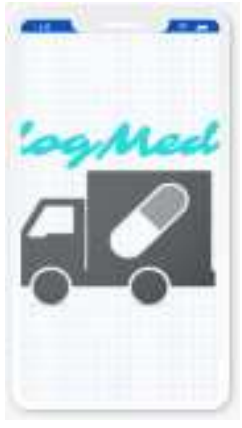

Figura 3.B. Ícone do menu inicial.

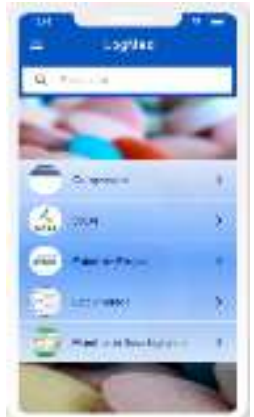

Fonte: Autores.

O botão "Comprasnet" (Figura 4.) contém um link que direciona o usuário, automaticamente, ao sistema de compras governamentais. O Comprasnet 4.0 é um sistema batizado assim, por se tratar de uma estrutura evolutiva do hoje conhecido Comprasnet. Uma alusão à Industria 4.0.

Atualmente o sistema conta com um escopo já ampliado das demais etapas do ciclo de vida da compra pública. Apesar de estar ainda em evolução, já conta com alguns novos subsistemas: planejamento institucional e anual de compras (PGC); primeiro módulo da fase interna e preparatória das licitações Estudo Técnico Preliminar (ETP Digital); gestão e fiscalização 
contratual (Comprasnet Contratos); nova sala de disputa de licitações para usuário fornecedor totalmente reestruturada e um aplicativo mobile que possibilita acesso facilitado a oportunidades de venda para o Governo.

Ao final, o ecossistema Comprasnet 4.0 deverá ser um sistema único e integrado, permitindo a operacionalização e controle de diversas etapas ao longo do ciclo de vida da compra pública. Será possível aos servidores públicos, gestores de governo, fornecedores, órgãos de controle e cidadãos interagirem entre si, no sistema, e com o sistema, extraindo dele, seu objetivo final.

Figura 4. Ícone do botão Comprasnet.

Fonte: Autores, adaptado de https://www.comprasnet.gov.br/seguro/loginPortal.asp (Comprasnet, [s.d.]).

O botão "SICAF" (Figura 5.) contém um link que direciona o usuário, automaticamente, ao sistema de Cadastramento Unificado de Fornecedores governamentais. O Sistema de Cadastramento Unificado de Fornecedores viabiliza o cadastramento de fornecedores de materiais e serviços para os órgãos e as entidades da Administração Pública Federal Direta, Autárquica e Fundacional, no âmbito do Sistema Integrado de Serviços Gerais - SISG. Uma vez inscrito no SICAF, o fornecedor estará cadastrado perante qualquer órgão/entidade que utiliza o SIASG.

Figura 5. Ícone do botão SICAF.

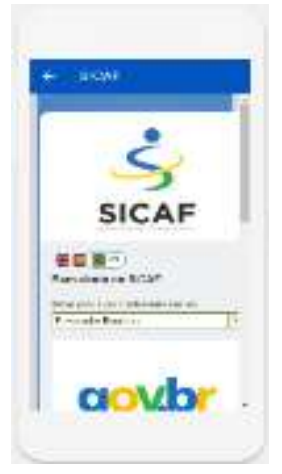

Fonte: Autores, adaptado de https://www.gov.br/compras/pt-br/sistemas/sicaf-digital (SICAF - Sistema de Cadastramento Unificado de Fornecedores).

O botão "Painel de Preços" (Figura 6.) contém um link que direciona o usuário, automaticamente, ao sistema de Cadastramento Unificado de Fornecedores governamentais. O Painel de Preços disponibiliza de forma clara e de fácil leitura, dados e informações de compras públicas homologadas no Sistema de Compras do Governo Federal - COMPRASNET. Tem como objetivo auxiliar os gestores públicos nas tomadas de decisões nas execuções de processos de compras, dar transparência em relação aos preços praticados pela Administração Pública e estimular o controle social. 
Figura 6. Ícone do botão Painel de Preços.

Fonte: Autores, adaptado de https://paineldeprecos.planejamento.gov.br (Painel de Preços, [s.d.]).

O botão "Documentos" (Figura 7.) é subdividido em seis abas consecutivas dispostas em: Rotina de recebimento de medicamentos, envio de nota de empenho, cobrança de carta de comprometimento de troca, notificação de procedimento administrativo, convocação do fornecedor para reunião e abertura de processo administrativo.

Figura 7. Aba de documentos.

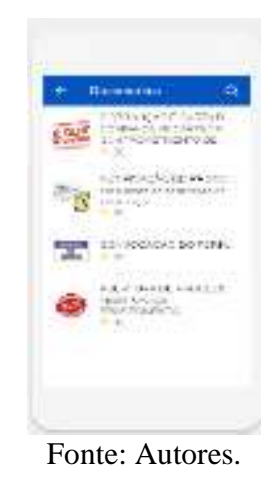

A opção denominada "Rotina de recebimento de medicamentos" (Figura 8.) contém um Procedimento Operacional Padrão para o recebimento de medicamentos na Central de Abastecimento Farmacêutico.

Figura 8. Ícone da aba Rotina de recebimento de Medicamentos.

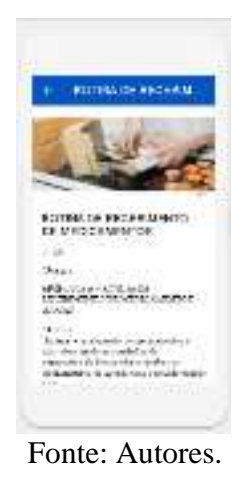

A opção denominada "Envio de nota de empenho" (Figura 9) contém um procedimento operacional padrão para o envio de nota de empenho da Central de Abastecimento Farmacêutico para o fornecedor. 
Figura 9. Ícone da aba Envio de Nota de Empenho.

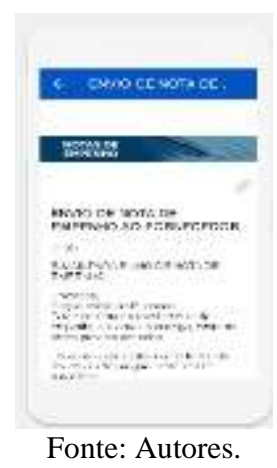

A opção denominada "Cobrança de carta de comprometimento de troca" (figura 10) contém um Procedimento Operacional Padrão para o Envio de Cobrança de carta de comprometimento de troca da Central de Abastecimento Farmacêutico para o fornecedor.

Figura 10. Ícone da aba Cobrança de Carta de Comprometimento de Troca.

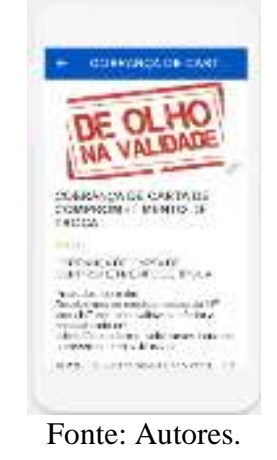

A opção denominada "Notificação de procedimento administrativo" (figura 11.) contém um procedimento operacional padrão para o envio de notificação de procedimento administrativo da Central de Abastecimento Farmacêutico para o fornecedor.

Figura 11. Ícone da aba Notificação de procedimento administrativo.

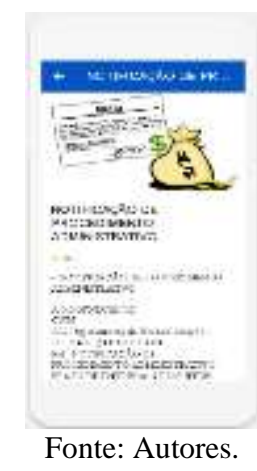

A opção denominada "Convocação do fornecedor para reunião"(figura 12.) contém um Procedimento Operacional Padrão para Convocação do fornecedor para reunião pela Central de Abastecimento Farmacêutico. 
Figura 12. Ícone da aba Convocação do Fornecedor para reunião.

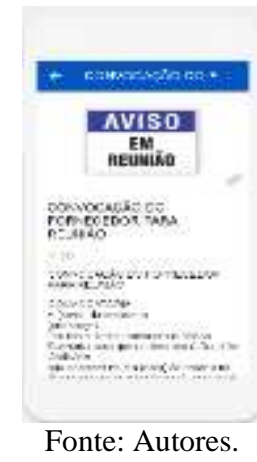

A opção denominada "Abertura de procedimento administrativo" (Figura 13.) contém um Procedimento Operacional Padrão para Abertura de procedimento administrativo da Central de Abastecimento Farmacêutico para o fornecedor.

Figura 13. Ícone da aba Abertura de procedimento administrativo.

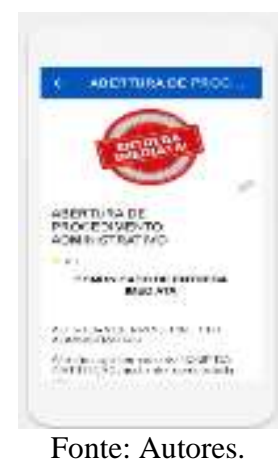

O botão "Planilha de fluxo logístico" (Figura 14.) contém um link https://docs.google.com/spread sheets/d/1ZqTz04Lkh7zUNkdDjr8a4eb7mBjiUGWC/edit\#gid=162386261(Fluxo Logístico 2019, [s.d.]) que direciona o usuário, automaticamente, para uma planilha online do google docs, a qual contém todos os dados logísticos das aquisições de medicamentos.

Figura 14. Ícone da aba Planilha de Fluxo logístico.

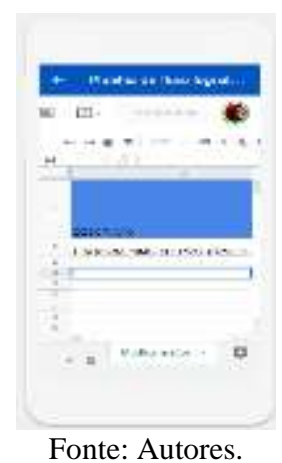

Os ícones foram determinados de maneira que se pudesse identificar os elementos descritos através das imagens e foram extraídos de forma online de fontes de domínio público.

A usabilidade do aplicativo foi avaliada por meio de um questionário elaborado pelo grupo de qualidade em software da Universidade Federal de Santa Catarina foi aplicado para a avaliação da usabilidade do protótipo (GQS/UFSC).O instrumento 
citado anteriormente refer-se a uma escala de usabilidade de aplicações de telefones com touch-screen através de uma única pontuação, sendo desenvolvido em duas etapas (Rosa \& Veras, 2013).

O questionário Match, que segue as dez heurísticas de Nielsen para avaliação de usabilidade. É composto de 48 questões, cada questão tem como alternativas as opções "SIM", "NÃO" e "NÃO SE APLICA". Ao término do preenchimento pelo desenvolvedor, os aplicativos são classificados, como mostrado na Figura 15.

Figura 15. Questionário MATcH.

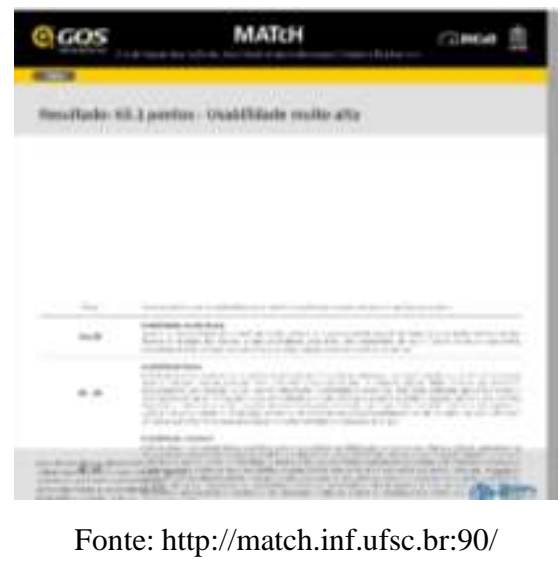

A validação da versão de teste do protótipo LogMed ocorreu em 19 de 06 de 2021. A análise foi realizada pelos próprios autores. Ao final da avaliação obteve-se a pontuação de 63,1 Pontos, classificação de usabilidade muito alta.

\section{Discussão}

Embora a irregularidade do abastecimento e a ausência de materiais sejam problemas frequentes em organizações de serviços públicos de saúde, a discussão sobre o processo logístico, abastecimento e cadeia de suprimentos é notadamente ausente na literatura nacional (Infante \& Santos, 2007).

Um dos processos mais difíceis de se otimizar é a logística de suprimentos hospitalares, especialmente no contexto dos medicamentos (Costa \& Oliveira, 1999).

A implantação de uma sistemática de qualificação e gestão dos fornecedores em âmbito institucional, de forma continuada, com critérios e instrumentos definidos, levaria ao aprimoramento dos serviços por eles prestados, além da seleção de possíveis parceiros comerciais. Os fornecedores deveriam obedecer às exigências técnicas e administrativas contidas no edital convocatório, além das especificações técnicas para a entrega dos medicamentos. De posse dos dados de gestão, o gestor poderia então adotar medidas corretivas, caso necessário, evitando a participação de fornecedores com desempenho abaixo do limite mínimo exigido em processos licitatórios futuros(Marin et al., 2003).

O Software para aplicações móveis, denominadas App's (aplicativo), impulsiona o interesse dos clientes, sendo uma ferramenta de pesquisa fidedigna que simplifica e acelera o atendimento. No entanto, para a eficácia dessa proposta, é fundamental que seu nível de usabilidade e credibilidade seja Elevado (F. G. F. Pereira et al., 2016).

Inclusive, há uma atenção de agências governamentais, empresas comerciais e organizações de saúde pública voltado para o uso dos aplicativos como instrumento na transformação dos hábitos de saúde, devido a plausíveis inferências efetivas dos aplicativos na saúde pública (Zhao et al., 2016). 


\section{Considerações Finais}

A aplicação da nova metodologia irá gerar um dossiê para acompanhamento da logística de entrega de medicamentos de cada fornecedor, visando otimizar esse processo e evitar o desabastecimento e seus consequentes prejuízos na assistência.

Uma ferramenta importante para o monitoramento do processo de aquisição pode ser a elaboração de relatórios mensais de gestão de fornecedores, que evidencie comportamento irregular do fornecedor ou ocorrências com produtos recebidos.

A partir das informações logísticas coletadas, o gestor poderá adotar as medidas corretivas necessárias e, até mesmo, evitar a participação em processos licitatórios futuros daqueles fornecedores que tenham um desempenho abaixo do limite mínimo exigido.

Torna-se importante salientar que recentemente foi sancionada a nova Lei de Licitações e Contratos Administrativos, LEI N ${ }^{o}$ 14.133, DE $1^{\circ}$ DE ABRIL DE 2021 (BRASIL,2021., [s.d.]). O novo marco legal substitui a Lei de Licitações (8.666/93), a Lei do Pregão (10.520/02) e o Regime Diferenciado de Contratações (RDC - Lei 12.462/11), além de agregar temas relacionados. Nos próximos 2 anos, os órgãos públicos poderão optar entre utilizar as normas antigas ou a nova lei. Ao final do biênio, ela passará a ser obrigatória para todos.

\section{Referências}

App LogMed. Https://galeria.fabricadeaplicativos.com.br/logmed?feature=facebook_storefront.

Brasil (1993). ([s.d.]). BRASIL, Presidência da República, Casa Civil, Subchefia para assuntos jurídicos - Lei 8.666, de 21/06/1993, que regulamenta o art. 37, inciso XXI, da Constituição Federal, institui normas para licitações e contratos da Administração Pública e dá outras providências. Brasília, DF, (1993). Http://www.planalto.gov.br/ccivil_03/Leis/L8666cons.htm.

Brasil (2002). ([s.d.]). Lei no 10.520, de 17 de julho de 2002. Institui, no âmbito da União, Estados, Distrito Federal e Municípios, nos termos do art. 37, inciso XXI, da Constituição Federal, modalidade de licitação denominada Pregão, para aquisição de bens e serviços comuns, e dá outras providências. Diário Oficial [da] República Federativa do Brasil, Poder 5 Executivo, Brasília, DF, 18 jul. 2002b. Http://www.presidencia.gov.br/ccivil_03/LEIS/2002/L10520.htm>.

Brasil (2006). (2006). Brasil. Ministério da Saúde. Secretaria de Ciência, Tecnologia e Insumos Estratégicos. Departamento de Assistência Farmacêutica e Insumos Estratégicos. Aquisição de medicamentos para a Assistência Farmacêutica no SUS. Brasília, 2006. Http://www. ensp.fiocruz.br/portalensp/judicializacao/pdfs/284.pdf.

Brasil (2021), I. LEI N 14.133, DE $1^{\circ}$ DE ABRIL DE 2021-DOU - Imprensa Nacional. Https://www.in.gov.br/web/dou.

Bridges, J. F. (2006). Lean systems approaches to health technology assessment. Pharmacoeconomics, 24(2), 101-109.

Comprasnet. Portal de Compras do Governo Federal. Https://www.gov.br/compras/pt-br/sistemas/comprasnet-siasg.

Costa, A. L., \& Oliveira, M. M. B. de. (1999). Sistema de informação para prescrição e distribuição de medicamentos: O caso do Hospital das Clínicas da Faculdade de Medicina de Ribeirão Preto. Revista de Administração-RAUSP, 34(3), 44-55.

Estrela, C. (2018). Metodologia científica: Ciência, ensino, pesquisa. Artes Médicas.

Fabapp—Fabrica de Aplicativos. ([s.d.]). Https://painel.fabricadeaplicativos.com.br/signin.

Fluxo Logístico 2019. ([s.d.]). Google Docs. Https://docs.google.com/spreadsheets/d/1QBsqrTkFKJDhzbg-i6r_F4Ov5ocopcaAwtIcnvRyZZU/edit?ouid=112 531419704250696890\&usp=sheets_home\&ths=true\&usp=embed_facebook.

Gonçalves, A. A., Yoshida, D., Pitassi, C., \& Freitas, J. A. de S. B. (2016). Fatores Críticos De Sucesso Na Gestão Da Cadeia De Suprimentos Em Saúde Pública. Uma Visão Dos Gestores Dos Institutos Federais Do Rio De JaneirO. RAHIS-Revista de Administração Hospitalar e Inovação em Saúde, 13(2).

Infante, M., \& Santos, M. A. B. dos. (2007). A organização do abastecimento do hospital público a partir da cadeia produtiva: Uma abordagem logística para a área de saúde. Ciência \& Saúde Coletiva, 12(4), 945-954.

Köche, J. C. ([s.d.]). José Carlos Köche Teoria da ciência e iniciação à pesquisa. http://webcache.googleusercontent.com/search?q =cache:s2QS3xFIi1gJ:btux.com.br/professorbruno/wp-content/uploads/sites/10/2018/07/K\%25C3\%25B6che-Jos\%25C3\%25A9-Carlos0D0AFundamentosde-metodologia-cient $\% 25 \mathrm{C} 3 \% 25 \mathrm{ADfica}$-_teoria-da0D0Aci\%25C3\%25AAncia-e-inicia\%25C3\%25A7\%25C3\%25A3o-\%25C3\%25A0pesquisa.pdf $+\& \mathrm{~cd}=1 \& \mathrm{hl}=\mathrm{pt}-\mathrm{BR} \& \mathrm{ct}=\mathrm{clnk} \& \mathrm{gl}=\mathrm{br}$.

Kumar, D., \& Seema. (2017). An analytical model for supplier selection. International Journal of Indian Culture and Business Management, 14(2), 131-140. LÜDKE, M. A. (2013). MEDA de. Pesquisa em educação: abordagens qualitativas. EPU.

Marin, N., Luiza, V. L., Osorio-de-Castro, C. G., \& Machado-dos-Santos, S. (2003). Assistência farmacêutica para gerentes municipais. In Assistência farmacêutica para gerentes municipais (p. 334-p). 
Research, Society and Development, v. 10, n. 8, e33510817363, 2021

(CC BY 4.0) | ISSN 2525-3409 | DOI: http://dx.doi.org/10.33448/rsd-v10i8.17363

Painel de Preços. Https://paineldeprecos.planejamento.gov.br/

Pereira, A. S., Shitsuka, D. M., Parreira, F. J., \& Shitsuka, R. (2018). Metodologia da pesquisa científica.[e-book]. Santa Maria. Ed. UAB/NTE/UFSM. Https://repositorio. ufsm. br/bitstream/handle ....

Pereira, F. G. F., Silva, D. V. da, Sousa, L. M. O. de, \& Frota, N. M. (2016). Building a digital application for teaching vital signs. Rev Gaúcha Enferm [Internet], 37(2), e59015.

Reis, A. M. M., \& Perini, E. (2008). Desabastecimento de medicamentos: Determinantes, consequiências e gerenciamento. Ciência \& Saúde Coletiva, 13(suppl), 603-610. https://doi.org/10.1590/S1413-81232008000700009.

Rosa, J. M., \& Veras, M. (2013). Avaliação heurística de usabilidade em jornais online: Estudo de caso em dois sites. Perspectivas em Ciência da Informação, $18(1), 138-157$.

SICAF - Sistema de Cadastramento Unificado de Fornecedores. Https://www3.comprasnet.gov.br/sicaf-web/index.jsf

Zhao, J., Freeman, B., \& Li, M. (2016). Can mobile phone apps influence people's health behavior change? An evidence review. Journal of medical Internet research, 18(11), e287. 